\title{
Concurrent bilateral ectopic pregnancy: a rarity
}

\author{
Prabhleen Kaur*, Urvashi Miglani, V. K. Kadam, Poonam Laul
}

Department of Obstetrics and Gynaecology, Deen Dayal Upadhyay Hospital, New Delhi, India

Received: 22 June 2015

Accepted: 22 July 2015

\section{*Correspondence:}

Dr. Prabhleen Kaur,

E-mail: dr.prabhleenahuja@gmail.com

Copyright: (c) the author(s), publisher and licensee Medip Academy. This is an open-access article distributed under the terms of the Creative Commons Attribution Non-Commercial License, which permits unrestricted non-commercial use, distribution, and reproduction in any medium, provided the original work is properly cited.

\begin{abstract}
Bilateral ectopic pregnancy is a rare twin gestation with only a few cases reported in the literature. We report a 30 year old woman without any high risk factor for ectopic pregnancy, who had concurrent bilateral ectopic pregnancy. A 30 year old female presented to the Gynecology emergency department complaining of vaginal bleeding and abdominal pain. The presumptive diagnosis of ruptured left sided ectopic pregnancy was made on basis of clinical findings and ultrasound finings. An emergency laparotomy was done revealed a hemoperitoneum of 1.5 liters, a ruptured left tubal pregnancy with active bleeding and right tubal un-ruptured ectopic was found. A bilateral salpingectomy was performed. Histopathology confirmed presence of chorionic villi in both tubes. In theory, laparoscopic salpingostomy is the best surgical approach in bilateral tubal pregnancy. However, bilateral salpingectomy may be necessary when both tubes are extensively damaged or are actively bleeding. Successful pregnancies have been reported after conservative surgical treatment of bilateral ectopic, but the risk of recurrence is high. Our decision for an emergency laparotomy followed by bilateral salpingectomy was based on the fact that the patient presented with acute abdomen and was haemodynamically unstable and there was extensive bilateral tubal damage. As the incidence of ectopic pregnancies is increasing concurrently with the incidences of pelvic inflammatory disease and use of assisted fertility techniques; it may be that these "rare ectopics" will become less uncommon.
\end{abstract}

Keywords: Concurrent; bilateral; Ectopic; Pregnancy

\section{INTRODUCTION}

Bilateral tubal ectopic pregnancy is the rarest forms of ectopic pregnancy. The fate of the two pregnancies is independent. Blastocyst normally implants in the endometrial lining of the uterine cavity. Implantation elsewhere is considered an ectopic pregnancy. The incidence of ectopic pregnancy has been reported to be increasing in recent years and common risk factors being assisted reproductive technique, pelvic infection and tubal surgery. Prior tubal damage confers the highest risk for ectopic pregnancy. ${ }^{1}$ Twin tubal pregnancy with both embryos in the same tube as well as with one in each tube has also been reported. The estimated incidence of bilateral tubal pregnancy is 1 in 725 to 1 in 1580 of all ectopic pregnancies. ${ }^{2,3}$ They are usually diagnosed at the time of surgery.

\section{CASE REPORT}

A 30 years old female, G3P2L2 with previous two full term home deliveries 10 and 9 years back respectively presented in emergency on $29^{\text {th }}$ April 2015 with vaginal spotting and intermittent lower abdominal pain for 2 days following amenorrhea of six weeks. Her previous cycles were regular and her personal and family history was unremarkable. On examination she was conscious and oriented having moderate pallor and tachycardia. Her pulse rate was $120 / \mathrm{min}$, blood pressure 90/60 in supine position; respiratory rate $24 / \mathrm{min}$. Abdomen was 
distended, tense and tender. Per vaginal examination showed pale vaginal mucosa, there was bleeding from cervical os, uterus was bulky and both fornices were extremely tender and cervical movement tenderness was noted .Her urine pregnancy test was positive, and serum beta-hCG 6887, Haemoglobin-7.4gm\%, trans-vaginal ultrasound revealed empty uterus with a heterogeneous mass of $5.3 \times 5.2 \mathrm{~cm}$ below and close to the left ovary and free fluid in peritoneal cavity. Blood grouping, cross matching was done and basic biochemical investigations were sent while she was being given supportive treatment of shock. The diagnosis of ruptured ectopic pregnancy was made on clinical findings and ultrasonography and patient was taken up for immediate laparotomy with arrangement of two units of blood. On Laparotomy the diagnosis of bilateral tubal ectopic was made (Figure 1). Hemoperitoneum approx. 1.5 liter was present, there was right sided un-ruptured ampullary ectopic gestation of 3 $\mathrm{X} 2 \mathrm{~cm}$ (Figure 2) and left sided ruptured isthmic ectopic gestation was found, uterus was normal in size and both ovaries were normal. Family was counseled about the intra-operative findings and written informed consent was taken for salpingectomy. Bilateral salpingectomy was done. Specimens were sent for histopathology. Two units of blood were transfused post operatively. Postoperative recovery was normal. On follow up, histopathology finding of both fallopian tubes revealed extensive hemorrhage, few well-formed villi and presence of decidua in the walls which confirmed the presence of bilateral ectopic pregnancy.

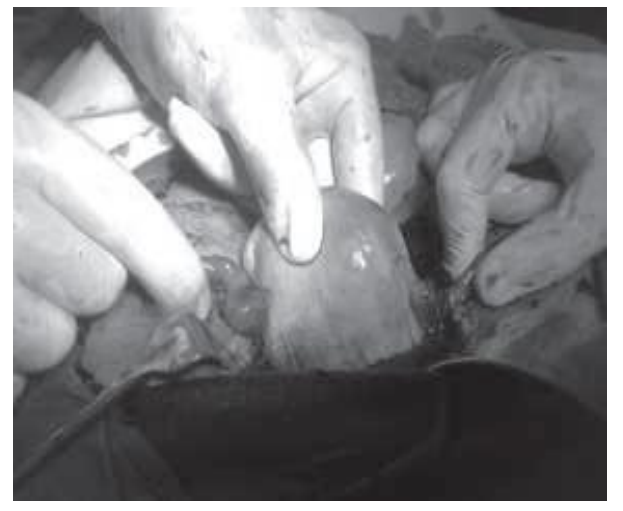

Figure 1: Bilateral tubal ectopic pregnancy.

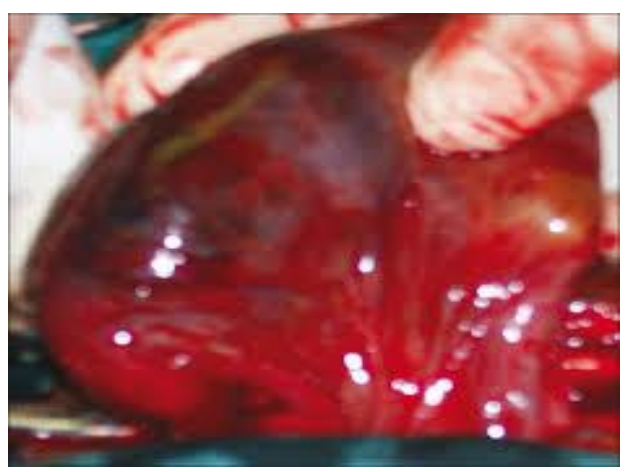

Figure 2: Right sided un-ruptured ampullary ectopic pregnancy.

\section{DISCUSSION}

Ruptured ectopic pregnancy is not a very uncommon diagnosis in emergency admissions; but concurrent bilateral tubal pregnancy is very rare. Complications of ectopic pregnancy like tubal or uterine rupture depending on the location of pregnancy which in turn can lead to massive hemorrhage, shock, disseminated intravascular coagulopathy and death are usually due to to misdiagnosis and late diagnosis. Hemorrhage from ectopic pregnancy is the leading cause of maternal death in the first trimester. ${ }^{4}$ The continuing development in all arenas of medical technology has allowed for easier and faster diagnosis of ectopic pregnancy, as well as improvement in the quality of treatment and outcome.

A review of the English language literature from 1965 to the present revealed no reported cases in which a preoperative diagnosis of bilateral ectopic pregnancy was made. All had a preoperative diagnosis of ectopic, presumed unilateral, made based on the $\beta$-hCG and transvaginal ultrasound. The most common method of diagnosing the second ectopic is by direct inspection of the contralateral tube intra-operatively. In all but two of the cases reviewed the diagnosis was made intraoperatively like our case. In the other two cases the second ectopic was diagnosed later when it became symptomatic. ${ }^{5}$

Some debate exists regarding what constitutes the definition of "bilateral ectopic pregnancy". In 1939 Fishback suggested criteria for the diagnosis of bilateral tubal ectopic pregnancy. The criteria required a description of the fetuses, or any portion of them, as well as a description of placental material. ${ }^{6}$ In 1953, Norris revised these criteria and broadened the definition, stating that the presence of chorionic villi in each tube should be sufficient to justify the diagnosis. ${ }^{7}$

Andrews $\mathrm{J}$ reported a case of spontaneous bilateral tubal pregnancy diagnosed intra-operatively. Conservative laparoscopic tubal surgery was used for treatment. The patient returned with signs of persistent ectopic pregnancy and subsequently treated with methotrexate. ${ }^{8}$ Martinez et al, reported a case of bilateral tubal ectopic where USG showed normal empty uterus with anechogenic images in left tube and in right tube. At laparoscopy right tube appeared as ectopic and left tube as hydrosalpinx. Bilateral salpingectomy was performed and pathology reports suggest bilateral tubal ectopic pregnancy. ${ }^{9}$ Himangini $B$ et al. reported a case of spontaneous bilateral tubal ectopic in which left tubal pregnancy was intact and right tubal ectopic was ruptured. Three quarter of both the tubes being damaged bilateral salpingectomy was done. ${ }^{10}$ Approximately 200 cases of bilateral tubal ectopic pregnancy have been reported in the literature to date. ${ }^{11}$

In theory, laparoscopic salpingostomy is the most appropriate and safest treatment. However, bilateral 
salpingectomy may be necessary when both tubes are extensively damaged or are actively bleeding. ${ }^{12}$ Our decision of an emergency laparotomy was due to patient being haemodynamically unstable. Although successful pregnancies have been reported after conservative surgical treatment of bilateral tubal pregnancy, the risk of recurrence is high. Our patient had ruptured left tube, abundant hemoperitoneum and right tube was unruptured but was extensively damaged by the mass and as patients family was complete so bilateral salpingectomy was done only after taking written informed consent. Bilateral ectopic was confirmed on histopathological examination.

\section{CONCLUSION}

Bilateral tubal pregnancy is the rarest form of ectopic pregnancy. The diagnosed of bilateral tubal pregnancy is usually made intra-operatively. Thus, this demonstrates the importance of identifying and closely examining both tubes at the time of surgery.

Funding: No funding sources Conflict of interest: None declared

Ethical approval: Not required

\section{REFERENCES}

1. Barnhart K, Sammel M, Gracia C. Risk factors for ectopic pregnancy in women with symptomatic firsttrimester pregnancies. Fertility and Sterility 2006;(1):36-43.

2. Basly M, Archour R, Aissa I. Extra-Uterine Twin Pregnancy. Case Report of Spontaneous Bilateral Tubal Ectopic Pregnancy. The Internet Journal of Gynaecology and Obstetrics. 2012;16(2):33-4.
3. Greenberg J. Bilateral Ectopic Pregnancy. Review Obstetrics Gynaecology. 2008;1(2):48.

4. Grimes D. Estimation of pregnancy-related morality risk by pregnancy outcome, United States 1991 to 1999. Am J Obstet Gynecol. 2006;194(1):22-4.

5. 92-94.Shenoy J, Choudhary V, Giles R. Bilateral ectopic pregnancy. J Obstet Gynaecology. 2005;25:612-3.

6. Fishback, H., Bilateral simultaneous tubal pregnancy. Canadian medical associated journal 1953;68(4):397-81.

7. Norris, S., Bilateral simultaneous tubal pregnancy. Canadian Medical Associate Journal. 1953;68: 37981.

8. Andrews J, Farrell S. Spontaneous bilateral tubal pregnancies: a case report. Journal of Obstetrics and Gynaecology Canada 2008;30(1):51-4.

9. Martinez J, Ana C, Gonzalez M, Gil O, Farrer M , Romero J. Bilateral Simultaneous Ectopic Pregnancy. Southern Medical Journal 2009;102(10):1055-7.

10. Himangini B, Behera R, Sachdev S. Spontaneous bilateral tubal ectopic pregnancy. Pravara Med Rev 2010;(1):19-21.

11. Ryan M, Saldana B: Bilateral ectopic pregnancy: A tale of caution. Acad Emerg Med 2000;7:1160-3.

12. Bangsgaard $\mathrm{N}$. Improved fertility following conservative surgical treatment of ectopic pregnancy. British Journal of Obstetrical and Gynaecology, 2003;110:765-70.

Cite this article as: Kaur P, Miglani U, Kadam VK, Laul P. Concurrent bilateral ectopic pregnancy: a rarity. Int J Reprod Contracept Obstet Gynecol 2015;4:1197-9. 